\title{
Impact of a Hospitalist-Run Procedure Service on Time to Paracentesis and Length of Stay
}

\author{
Evan Ritter, MD*, Manpreet Malik, MD², Rehan Qayyum, MD, MHS, SFHM¹
}

'Division of Hospital Medicine, Virginia Commonwealth University School of Medicine, Richmond, Virginia; ${ }^{2}$ Division of Hospital Medicine, Emory University School of Medicine, Atlanta, Georgia.

Hospitals are establishing procedure services to address resident training and patient safety. We examined whether a hospitalist procedure service affects a patient's hospital length of stay (LOS) and the time from admission to paracentesis (A2P). We queried our electronic medical records for all inpatient peritoneal fluid samples from July 1, 2016, to May 31, 2019. LOS and A2P time were compared among patients who had paracentesis by the procedure service, by residents, or by radiology. Of the 1,321 procedures, 509 (38.5\%) were performed by the procedure service. In the adjusted analysis, as compared with procedure service, the group that underwent paracentesis by the radiology service had a $27 \%$ longer LOS $(95 \% \mathrm{Cl}, 2 \%-58 \%)$ and $40 \%$ longer A2P time $(95 \%$ $\mathrm{Cl}, 5 \%-87 \%)$. The resident group had shorter A2P (-19\%; $95 \% \mathrm{Cl},-33 \%$ to $0.2 \% ; P=.05$ ) than the procedure service group but similar LOS. To our knowledge, this is the first study that suggests patient-centered benefits of a hospitalist procedure service. Journal of Hospital Medicine 2021;16:476-479. () Society of Hospital Medicine

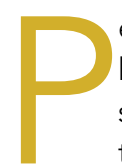

eritoneal fluid examination is often recommended for hospitalized patients with ascites. ${ }^{1}$ The prevalence of spontaneous bacterial peritonitis (SBP) in these patients ranges from $10 \%$ to $30 \% .^{2-6}$ Bedside paracentesis has clinical outcomes similar to that performed by radiology, with an improved length of stay (LOS) and decreased transfusion requirements.?

Internal medicine residency programs are establishing procedure services to address concerns about resident training in procedures and patient safety. Previous studies, which include paracentesis in patients with cirrhosis, have focused on resident comfort with procedures, supervision, procedural complications, and patient satisfaction. ${ }^{8-12}$ However, the impact of a procedure service on the time from admission to the procedure has not been studied. In this study, we aimed to examine whether the institution of a hospitalist-run procedure service affected a patient's LOS in the hospital and the time difference between a patient's hospital admission and paracentesis (A2P).

\section{METHODS}

An inpatient hospitalist-run procedure service was introduced on July 1, 2016. The service was staffed by a hospitalist and second-year internal medicine residents. The service is available 7:00 AM to 5:00 PM all days of the week. To identify patients who underwent paracentesis, we queried our electronic medical re-

*Corresponding Author: Evan Ritter, MD; Email: evan.ritter@vcuhealth.org; Telephone: 804-827-1081; Twitter: @ERitterMD.

Published online first May 19, 2021.

Received: January 8, 2020; Revised: October 30, 2020;

Accepted: December 8, 2020

๑) 2021 Society of Hospital Medicine DOI 10.12788/jhm.3582 cords for all peritoneal fluid samples from July 1, 2016, to May 31 , 2019. Paracenteses performed in the outpatient clinics, in the radiology suite, or in the emergency department were excluded if the patient was not admitted. We also excluded patients who had paracentesis within 6 hours of presentation, as these patients likely had an urgent clinical indication for paracentesis.

Data on age, gender, race, ethnicity, date and time of hospital admission, and discharge date and time were retrieved. We also retrieved data on the absolute number of polymorphonuclear leukocytes (PMN) in the peritoneal fluid sample; a patient with a count higher than 250/uL was considered to have SBP. The timestamp for the peritoneal fluid results was used to approximate the A2P time. Paracenteses performed by or under direct supervision of procedure service hospitalists were identified through a procedure log maintained by procedure service hospitalists. We generated a binary variable to differentiate patients who were admitted during the day from those admitted during the night, when the procedure service was not available. For all patients, we calculated the model for end-stage liver disease and sodium (MELD-Na) score. ${ }^{13}$ Groups performing paracenteses were categorized into procedure service, residents, and radiology. Primary clinical services were categorized into general medicine, gastroenterology, surgery, and others.

Data were summarized as mean (SD) or median (interquartile range) for continuous variables and as percentages for categorical variables. Patients who had paracenteses by radiology or residents during the study period were considered controls. We used concurrent controls to address secular time trends (eg, measures to decrease LOS or changes in ordering tests in the electronic health record) in outcome measures. Patient characteristics were compared using the Wilcoxon rank-sum test or the $\chi^{2}$ test, as appropriate. 
TABLE 1. Study Population Characteristics

\begin{tabular}{|c|c|c|c|}
\hline Variable & $\begin{array}{l}\text { Procedure service } \\
\quad(\mathrm{N}=509)\end{array}$ & $\begin{array}{c}\text { Other } \\
(\mathrm{N}=812)\end{array}$ & $P$ value \\
\hline Age, y & $55.8(11.1)$ & $55.0(12.9)$ & 0.47 \\
\hline Female, No. (\%) & $187(36.7)$ & $315(38.8)$ & .45 \\
\hline White, №. (\%) & $304(59.7)$ & $424(52.2)$ & .008 \\
\hline Polymorphonuclear cells, cells/ $/ \mathrm{L}^{\mathrm{a}}$ & $95(632)$ & $264(2801)$ & .83 \\
\hline Length of stay, median (IQR), h & $174(251)$ & $223(443)$ & $<.001$ \\
\hline A2P, median (IQR), h & $43(101)$ & $52(165)$ & .03 \\
\hline Spontaneous bacterial peritonitis, No. (\%) & $23(4.5)$ & $38(4.7)$ & .90 \\
\hline DRG weights, median (IQR) & $1.7(0.8)$ & $1.8(2.6)$ & $<.001$ \\
\hline MELD-Na score & $21.6(8.6)$ & $21.9(9.1)$ & .84 \\
\hline INR & $1.7(0.61)$ & $1.7(0.83)$ & .002 \\
\hline Platelet count, $10^{9} / \mathrm{L}$ & $167(143)$ & $195(126)$ & $<.001$ \\
\hline Cirrhosis, No. (\%) & $419(82.3)$ & $444(54.7)$ & $<.001$ \\
\hline Day (7:00 AM - 5:00 pM), №. (\%) & $260(51)$ & $408(50)$ & .77 \\
\hline Primary service & & & $<.001$ \\
\hline General medicine, №. (\%) & $226(44)$ & $307(38)$ & \\
\hline Gastroenterology, №. (\%) & $190(37)$ & $151(19)$ & \\
\hline Surgery, No. (\%) & $19(3.7)$ & $150(18)$ & \\
\hline Others, No. (\%) & $74(15$ & $204(25)$ & \\
\hline
\end{tabular}

Data are presented as mean (SD) unless noted otherwise. $P$ values were calculated using the Wilcoxon rank-sum test or $\chi^{2}$ test.

a Both the median and IQR were 0 for both groups.

Abbreviations: A2P, admission to paracentesis; DRG, diagnosis-related group; INR, international normalized ratio; IQR, interquartile range; MELD-na, model for end-stage liver disease and sodium.

Two outcome variables were examined: LOS, and A2P time. Because both outcome variables were right skewed, we used generalized linear models with gamma distribution and log link. The advantage of a generalized linear model approach is that the transformed coefficients are better interpretable than when using the log transformation of the response variable. ${ }^{14}$ To account for time trends, we included time in months in the model. Models were adjusted for age, gender, race, whether the admission was during day or night, PMN in peritoneal fluid, MELD-Na score, platelet count on the day of procedure, presence or absence of cirrhosis, diagnosis-related groups weight, primary clinical service, and the group performing paracentesis. To address heterogeneity among patients included in our study and the fact that some patients had multiple paracenteses, we conducted sensitivity analyses by excluding all noncirrhotic patients and including only the first paracentesis. A $P$ value less than .05 was considered significant. All statistical analyses were performed using Stata MP 16.0 for Windows (StataCorp LLC).

\section{RESULTS}

Of the 1,321 paracenteses included in our study, 509 (38.5\%) were performed by the procedure service, 723 (54.7\%) by residents, and 89 (6.7\%) by radiology. For comparison, $15.4 \%$ of procedures were performed by the radiology service during the 3 years before the start of the procedure service. More than $50 \%$ of the first paracenteses were performed within 30 hours of admission. Hospitalists or residents under the direct supervision of a hospitalist performed all paracenteses. Residents performing paracenteses, when not on the procedure service, were on general internal medicine, gastroenterology, hematology and oncology, or surgical services. No failed paracentesis attempts by the procedure service were subsequently performed by radiology. The mean age of the participants was 55.3 (12.2) years, 728 (55\%) were White, 502 (38\%) were female, and SBP was present in 61 (4.6\%) patients. There was no difference by age, gender, time of admission, presence of $\mathrm{SBP}$, or peritoneal fluid PMN in patients who underwent paracentesis by the procedure service versus controls (Table 1). A higher proportion of White patients and patients with cirrhosis underwent paracentesis by the procedure service than by another service. The LOS and A2P time were significantly lower for patients who underwent paracentesis by the procedure service than by another service (Table 1). When examining the adjusted linear secular time trends, LOS decreased by $0.1 \%$ per month $(95 \% \mathrm{Cl},-0.5 \%$ to $0.8 \% ; P=.67)$ and $\mathrm{A} 2 \mathrm{P}$ time by $0.02 \%$ per month $(95 \% \mathrm{Cl},-1.0 \%$ to $1.1 \% ; P=.96)$.

In unadjusted models but accounting for secular time trends, patients who had paracenteses performed by residents or by radiology had a $50 \%(95 \% \mathrm{Cl}, 22 \%-83 \% ; P=.002)$ and $127 \%$ (95\% Cl, 65\%-211\%; $P<.001)$ longer LOS, respectively, than when paracentesis was performed by the procedure service. After adjusting for potential confounders, the difference in LOS between radiology and the procedure service remained significant; patients who had a paracentesis performed by radiology had a $27 \%(95 \% \mathrm{Cl}, 2 \%-58 \% ; P=.03)$ longer LOS than patients who had the procedure performed by the procedure service. This relative LOS translates into $88(95 \% \mathrm{Cl}, 1-174$ hours) additional hours in absolute LOS. There was no difference in LOS between the procedure service and residents in the adjusted analysis (Table 2).

Similarly, in unadjusted models for A2P time and accounting for secular time trends, patients who had a paracentesis performed by residents or by radiology had a $52 \%$ (95\% Cl, 23\%$88 \% ; P<.001)$ and $173 \%(95 \% \mathrm{Cl}, 109 \%-280 \% ; P<.001)$ longer A2P time, respectively, than patients whose paracentesis was performed by the procedure service. After adjusting for potential confounders, the difference in A2P time between radiology and the procedure service remained significant. Patients who had paracentesis performed by radiology had a 40\% (95\% $\mathrm{Cl}, 5 \%-87 \% ; P=.02)$ longer $\mathrm{A} 2 \mathrm{P}$ time than patients who had paracentesis performed by the procedure service. This relative increase translates into 52 (95\% Cl, 3.3-101 hours) additional hours in absolute A2P time. On the other hand, residents had a significantly shorter $\mathrm{A} 2 \mathrm{P}$ time $(-19 \%, 95 \% \mathrm{Cl},-33 \%$ to $0.2 \%$; $P=.05)$ (Table 2). 
TABLE 2. Effect of Procedure Service on Length of Stay and Time From Admission to Procedure

\begin{tabular}{|c|c|c|c|c|}
\hline & \multicolumn{2}{|c|}{ Length of stay } & \multicolumn{2}{|c|}{ Admission to procedure time } \\
\hline & Relative change $(95 \% \mathrm{Cl}), \%$ & $P$ value & Relative change $(95 \% \mathrm{Cl}), \%$ & $P$ value \\
\hline \multicolumn{5}{|l|}{ Unadjusted models ${ }^{a}$} \\
\hline Procedure service & Reference & & Reference & \\
\hline Residents & $50(22-83)$ & .002 & $52(23-88)$ & $<.001$ \\
\hline Radiology & $127(65-211)$ & $<.001$ & $173(97-280)$ & $<.001$ \\
\hline \multicolumn{5}{|l|}{ Adjusted models } \\
\hline Procedure service & Reference & & Reference & \\
\hline Residents & $-1(-14$ to 13$)$ & .85 & $-19(-33$ to 0.2$)$ & .05 \\
\hline Radiology & $27(2-58)$ & .03 & $40(5-87)$ & .02 \\
\hline \multicolumn{5}{|c|}{ Sensitivity analysis, adjusted } \\
\hline Procedure service & Reference & & Reference & \\
\hline Residents & 5 (-10 to 22$)$ & .51 & $-19(-36$ to 3$)$ & .08 \\
\hline Radiology & 47 (4 to 208) & .03 & $91(19-207)$ & .008 \\
\hline
\end{tabular}

In the sensitivity analysis, excluding noncirrhotic patients and including only the first paracentesis for patients who had multiple procedures performed during admission, the results remained unchanged. In adjusted analysis, patients who had paracentesis performed by radiology had a $47 \%(95 \% \mathrm{Cl}$, $3.7 \%-108 \% ; P=.03$ ) longer LOS and $91 \%$ (95\% Cl, 19\%-107\%; $P=.008)$ longer A2P time than when paracentesis was performed by the procedure service. There were no differences in LOS or A2P time between the procedure service and residents (Table 2).

\section{DISCUSSION}

In this study, we report that a hospitalist-run procedure service, when compared with a radiology service, is associated with decreased LOS and A2P time independent of studied potential confounders and secular time trends. We also showed that, compared with radiology, the A2P time for nonemergent procedures (those performed 6 hours after admission) was not adversely affected by the procedure service. Residents performing paracenteses independently had shorter A2P time than the procedure service.

Although several institutions have bedside procedure services, data are lacking on benefits. Previously, paracenteses performed by residents have been associated with decreased LOS and need for transfusions when compared with radiology. ${ }^{7}$ Our study extends these findings to show a shortened A2P time. Delays may occur when a patient is referred to radiology because of volume, triaging of higher-acuity procedures, and transportation. Procedure services provide consistent attending supervision, more procedures by upper-level residents, and a lower rate of unsuccessful procedures. ${ }^{12,15}$ Current study findings support the importance of continuing bedside procedure training for at least those residents who are interested in hospital medicine. ${ }^{7}$

Our study has several strengths and some potential limitations. The study examined outcomes that are important to patients as well as hospital administrators; it also had a large sample size, spanning 3 years. As it was a retrospective cohort study, there is potential for residual confounding due to unmeasured confounders. We did not examine the potential effect modification of procedure urgency, as such data are difficult to discern. Our method of identification missed patients who received therapeutic paracentesis without laboratory analysis. It is unclear why more White patients were referred to the procedure team; this is an area for further evaluation. Results of this study are likely not generalizable to institutions with a robust radiology service that has built-in redundancy to accommodate urgent procedures and easy availability over the weekends.

\section{CONCLUSION}

We found that a hospitalist-run teaching procedure service is associated with shorter LOS and A2P time. Further research is needed to determine if the benefits of a procedure service extend to lowering morbidity and/or mortality, as well as to determine the cost-effectiveness of a procedure service and whether the significant investment by the institution in establishing a procedure service is mitigated by the gains from better patient outcomes and reduced LOS.

Disclosures: The authors have no conflicts to disclose. 


\section{References}

1. Runyon BA. AASLD guidelines. Management of adult patients with ascites due to cirrhosis: update 2012. April 2013. https://www.aasld.org/sites/ default/files/2019-06/AASLDPracticeGuidelineAsciteDuetoCirrhosis Update2012Edition4.pdf

2. Rimola A, García-Tsao G, Navasa M, et al. Diagnosis, treatment and prophy axis of spontaneous bacterial peritonitis: a consensus document. International Ascites Club. J Hepatol. 2000;32(1):142-153. https://doi.org/10.1016/ S0168-8278(00)80201-9

3. Sort $P$, Navasa $M$, Arroyo $V$, et al. Effect of intravenous albumin on renal impairment and mortality in patients with cirrhosis and spontaneous bacterial peritonitis. N Engl J Med. 1999;341(6):403-409. https://doi.org/10.1056/ NEJM199908053410603

4. Gaetano JN, Micic D, Aronsohn A, et al. The benefit of paracentesis on hospitalized adults with cirrhosis and ascites. J Gastroenterol Hepatol. 2016:31(5):1025-1030. https://doi.org/10.1111/jgh.13255

5. Kim JJ, Tsukamoto MM, Mathur AK, et al. Delayed paracentesis is associ ated with increased in-hospital mortality in patients with spontaneous bacterial peritonitis. Am J Gastroenterol. 2014;109(9):1436-1442. https://doi. org/10.1038/ajg.2014.212

6. Chinnock B, Afarian H, Minnigan H, Butler J, Hendey GW. Physician clinical impression does not rule out spontaneous bacterial peritonitis in patients undergoing emergency department paracentesis. Ann Emerg Med. 2008;52(3):268-273. https://doi.org/10.1016/j.annemergmed.2008.02.016

7. Barsuk JH, Cohen ER, Feinglass J, McGaghie WC, Wayne DB. Clinical outcomes after bedside and interventional radiology paracentesis procedures. Am J Med. 2013;126(4):349-356. https://doi.org/10.1016/j. amjmed.2012.09.016
8. Huang GC, Smith CC, Gordon CE, et al. Beyond the comfort zone: residents assess their comfort performing inpatient medical procedures. Am J Med. 2006;119(1):71.e17-24. https://doi.org/10.1016/j.amjmed.2005.08.007

9. Lenhard A, Moallem M, Marrie RA, Becker J, Garland A. An intervention to improve procedure education for internal medicine residents. J Gen Intern Med. 2008;23(3):288-293. https://doi.org/10.1007/s11606-008-0513-4

10. Mourad M, Kohlwes J, Maselli J, MERN Group, Auerbach AD. Supervising the supervisors-procedural training and supervision in internal medicine residency. J Gen Intern Med. 2010;25(4):351-356. https://doi.org/10.1007/ s11606-009-1226-z

11. Mourad M, Auerbach AD, Maselli J, Sliwka D. Patient satisfaction with a hospitalist procedure service: is bedside procedure teaching reassuring to patients? J Hosp Med. 2011;6(4):219-224. https://doi.org/10.1002/jhm.856

12. Tukey $\mathrm{MH}$, Wiener RS. The impact of a medical procedure service on patient safety, procedure quality and resident training opportunities. J Gen Intern Med. 2014;29(3):485-490. https://doi.org/10.1007/s11606013-2709-5

13. Kim WR, Biggins SW, Kremers WK, et al. Hyponatremia and mortality among patients on the liver-transplant waiting list. N Engl J Med. 2008:359(10):10181026. https://doi.org/10.1056/NEJMoa0801209

14. Lindsey JK, Jones B. Choosing among generalized linear models applied to medical data. Stat Med. 1998;17(1):59-68. https://doi.org/10.1002/(sici)10970258(19980115)17:1<59::aid-sim733>3.0.co;2-7

15. Miller R, Garber A, Smith H, Malik M, Kimberly C, Qayyum R. Volume and supervision of resident procedures logged after implementation of a procedure medicine curriculum. J Gen Intern Med. Published online March 17 2020. https://doi.org/10.1007/s11606-020-05763-9

Journal of Hospital Medicine

Leading
By Exam

journalofhospitalmedicine.com

\#HowWeHospitalist 